\title{
Caractérisation du système sectoriel d'innovation du cacao au Cameroun
}

\section{Characterization of the sectoral innovation system for cocoa in}

\section{Cameroon}

\author{
Syndhia Mathée $e^{\star 1,2,4}$, Eric Joël Fofiri Nzossie ${ }^{3}$, Ludovic Temple ${ }^{1,4}$ \\ ${ }^{1}$ Cirad, UMR Innovation, F-34398 Montpellier, France syndhia.mathe@cirad.fr, ludovic.temple@cirad.fr \\ ${ }^{2}$ IITA-Cameroon, 1st, Main Road IRAD, Nkolbisson, PO Box 2008 (Messa), Yaoundé, Cameroon \\ ${ }^{3}$ Université de Ngaoundéré, Département de Geographie / Chercheur associé UMR 201 "Développement \& Sociétés" \\ IRD-IEDES, BP 320, Yaoundé (Cameroun) fofiri_eric@yahoo.fr \\ ${ }^{4}$ INNOVATION, University of Montpellier, CIRAD, INRAE, Institut Agro, Montpellier, France \\ Auteur correspondant
}

\begin{abstract}
RÉSUMÉ. L'article interroge en quoi le concept de Système Sectoriel d'Innovation (SSI) permet de comprendre et d'organiser des choix de politiques publiques sur la filière cacao au Cameroun. Cette production centrale dans l'économie agricole du pays, a fait l'objet depuis 2002 de multiple "plans de relance " visant à accroitre significativement les rendements et la production de cacao grâce au transfert des connaissances scientifiques et techniques. A partir d'entretiens ouverts et de collecte de données quantitatives auprès d'acteurs clés, l'article caractérise le mode et la logique d'organisation du " système cacaoyer d'innovation ". L'analyse de la structuration de ce SSI révèle des dysfonctionnements. Elle permet d'identifier les leviers potentiels pour une trajectoire d'innovation vers une augmentation durable et plus inclusive de la production.

ABSTRACT. This article examines how a Sectorial System of Innovation (SSI) can be used to understand and organize public policy choices in the cocoa sector in Cameroon. This central production in the country's agricultural economy has been, since 2002, the subject of several "recovery plans" aiming to significantly increase cocoa yields, and thus the production of cocoa, by sharing scientific and technical knowledge. Based on open interviews and quantitative data collected from key actors, this article characterizes the organizational mode and logic of the "cocoa innovation system". The analysis of the structuring of this SSI reveals problems. It identifies potential levers for an innovation trajectory towards a sustainable and more inclusive increase in cocoa production.

MOTS-CLÉS. Système Sectoriel d'Innovation, Politique de Recherche et d'Innovation, Cacao, Cameroun.

KEYWORDS. Sectorial System of Innovation, Research and Innovation policy, Cocoa, Cameroon.
\end{abstract}

JEL: 03, 031, 032, 033, 038

\section{Introduction}

Introduite dès le $\mathrm{XVI}^{\mathrm{e}}$ siècle en Afrique par les Espagnols, la cacaoyère camerounaise est l'une des plus anciennes du continent africain [ALA 96]. Plus de 600000 Exploitations Familiales Agricoles (EFA) participent à la production. Outre son rôle de marqueur de l'appropriation foncière en milieu rural [LEP 95], le cacaoyer est de plus en plus un lieu de placements de l'épargne pour sécuriser des revenus de retraite pour les populations urbaines.

La crise cyclique des cours du cacao dans un contexte de désengagement de l'Etat à partir des années 90 a été en faveur d'un accroissement de la production au détriement de la qualité. Elle s'est traduite par une récession de la production et des pertes de parts de marché amenant le Cameroun aujourd'hui a un plafond de verre autour des 300000 tonnes. La décennie 2000 a cependant connu une reprise des cours mondiaux qui relance la production et l'engagement de l'Etat dans le secteur. Le Cameroun est le 5ème producteur mondial de cacao avec une production qui oscille entre 250000 et de 290000 tonnes d'une année à l'autre [LES 19]. La production qui couvre 672745 ha 
$\left(\right.$ FAOSTAT $^{1}$ ) se concentre dans les régions du Centre, du Sud et du Sud-Ouest. La production est réalisée à près de $90 \%$ par des petits producteurs de 1,5 à 3 ha. Le système de culture est principalement basé sur des systèmes agroforestiers. Ces systèmes sont très peu intensifiés avec des rendements de $350 \mathrm{~kg} / \mathrm{ha}$. La production est principalement exportée en fèves. Face aux tendances actuelles de croissance de la demande mondiale en cacao, le Cameroun s'était fixé un objectif de 600.000 tonnes en 2020 qui n'a pas été atteint. Le nouveau Document de Stratégie nationale de développement 2020-2030 (SND30) fixe cette filière comme prioritaire et un objectif de doublement des rendements pour atteindre $730 \mathrm{~kg}$ en $2030^{2}$ [REP 21]. Il mobilise un ensemble d'investissements et de propositions techniques basés sur la volonté de moderniser les pratiques et les exploitations cacaoyères, en les accompagnant vers l'innovation principalement par le renouvellement du matériel végétal, le rajeunissement des vergers cacaoyers et la mise en place des Bonnes Pratiques Agricoles (BPA).

Ce contexte interroge comment analyser et accompagner les politiques de recherche et d'innovation pour un accroissement durable de la production, c'est-à-dire en diminuant la déforestation [CAR 20], en limitant l'usage de pesticides et en augmentant les revenus des petits producteurs. [CAR 20] recommande l'introduction des enjeux de durabilité envrionnementale dans l'action publique pour le développement du secteur cacao. Nous portons un regard sur les implications de cette recommendatation par rapport à l'organisation des acteurs qui accompagnent l'innovation dans le secteur cacao au Cameroun. Pour cela cette contribution propose de mobiliser un cadre d'analyse en termes de Système Sectoriel d'Innovation. La première partie caractérise comment ce cadre d'analyse permet d'identifier la structure de l'environnement dans lequel naissent et évoluent différentes innovations liées au cacao. Elle expose aussi le dispositif de collecte et de traitement des informations. La seconde partie présente les résultats en termes d'identification des dynamiques issues de l'organisation de ce système. La troisième discute les résultats en termes de politiques de recherche et d'innovation.

\section{Cadre méthodologique pour analyser l'information dans le secteur cacaoyer}

\subsection{Le cadre d'analyse Système Sectoriel d'Innovation (SSI)}

La notion de SSI et de production émerge dans les années 1990 [BRE 97 ; GEE 04]. Dans le secteur agricole et alimentaire, ce cadre d'analyse a été utilisé pour analyser les déterminants des changements technologiques dans l'industrie ou les biotechnologies [HAL 05]. Son utilisation est plus récente dans l'agriculture et l'agro-alimentaire où il rend compte de différentes communautés d'usages possibles [TOU 14] et où sont interrogées les spécificités des processus d'innovation liées à la nature même de la production agricole ou de processus technologiques en fonction des filières et des contextes macro-institutionnels, entre pays de l'OCDE et pays en développement [CAS 17]. La délimitation d'un SSI en relation avec ces travaux conduits à repérer trois composantes qui interagissent entre elles et se spécifient autour d'une filière ou d'un groupe de produits, ce sont des connaissances et technologies, un système acteur fonctionnant en réseau et des institutions qui régulent les relations entre acteurs [MAL 05].

$\mathrm{Au}$ regard de ce référentiel nous délimitons les contours d'un SSI comme un mille-feuille d'acteurs issus des sphères productive et institutionnelle ${ }^{3}$ qui se coordonnent pour générer, adopter

1 http://www.fao.org/faostat/fr/\#data/QC

2 Il est articulé autour de huit piliers : la recherche, la production, la commercialisation, la promotion et la consommation, la qualité, la transformation, le financement et les mesures d'accompagnement.

3 Sphère productive : utilisateurs, producteurs et fournisseurs d'intrants.

Sphère institutionnelle : universités, institutions financières, organismes gouvernementaux et ONGs. 
et utiliser de nouvelles technologies, nouveaux intrants (variétés, bio-intrants), ou nouveaux produits transformés à partir du cacao.

Les connaissances et technologies sont composées des connaissances de base, inputs et technologies spécifiques à un secteur. L'analyse de ces éléments requière la spécification des frontières du secteur. Les acteurs et les réseaux sont composés d'agents hétérogènes qui peuvent être des entreprises ou d'autres organisations (instituts de recherche, université, institutions de financement, coopératives, syndicats...). Les interrelations à travers des réseaux formels ou informels entre ces agents peuvent être marchandes ou non marchandes. Ces agents et les réseaux dont ils font partie sont des éléments déterminants permettant d'identifier les frontières du système. Les institutions incluent les normes sociales, routines, habitudes, pratiques, règles, loi et standards qui influent les comportements et les interactions des agents. Elles peuvent donc être formelles ou informelles et se déployer à différentes échelles spatiales. La dynamique des SSI est liée à deux grands mécanismes : un processus de création (produits, technologies, organisations, institutions, comportements, stratégies) et de sélection par le marché ou d'autres formes de coordination horsmarché (contrats, réciprocité, coopération) qui réduit l'hétérogénéité au sein du système.

\subsection{Dispositif de collecte de l'information et bases de données}

Le dispositif de collecte d'information vise à identifier le mode et la logique d'organisation des institutions et organisations dans le secteur du cacao. Il combine plusieurs sources (entretiens ouverts, base de mobilisation des données sur la distribution du matériel végétal amélioré, base de données bibliographiques sur les thèmes de recherche au Cameroun). Les entretiens ouverts ont été menés auprès d'organisations actrices du SSI, principalement : l'Office National du Cacao \& Café (ONCC), la Société de Développement du Cacao (SODECAO), le Conseil Interprofessionnel du Cacao \& Café (CICC), le Groupement des Exportateurs du Cacao \& Café (GEX), le Fonds de Développement des filières Cacao \& Café (FODECC), les Ministères de l'agriculture, du commerce et de la recherche, ainsi que les Sociétés industrielles des cacaos (SIC-CACAO) et les Chocolateries et confiseries du Cameroun (CHOCOCAM).

Les entretiens ont ciblé au sein de ces structures les personnes clés participant directement à la structuration du système d'innovation. Les entretiens avaient pour objet de révéler les stratégies d'action des acteurs et les connexions entre ces stratégies polarisées par la production, l'échange la transformation du cacao. Différentes bases de données ont été mobilisées auprès de différents acteurs interrogés sur la distribution de plants améliorés. Sur la base de la régularité temporelle des données disponibles, nous avons ainsi utilisé les bases de données de la SODECAO, du MINADER et de l'IITA ${ }^{4}$ comportant des variables comparables (années et localités d'intervention). Les données considérées couvrent la période 2009-2013. Elles ont permis de construire une représentation spatiale de la distribution de plants améliorés à l'aide de la combinaison des logiciels Adobe Illustrator et Philcarto. Ces données sont considérées comme un proxy des niveaux d'utilisation des variétés améliorées par les agriculteurs. Nous avons également utilisé une base de données bibliométriques sur le Web of science (1991-2013) constituée dans une étude sur l'économie de la recherche au Cameroun [BIS 14]. Cette base contient plus de 7000 publications scientifiques publiées par des chercheurs et organismes basés au Cameroun dans laquelle une extraction des publications spécifiques au cacao a été faite sur 411 références. Ces références révèlent une diversification des connaissances scientifiques sur le cacao au cours des dernières décennies. Enfin les auteurs mobilisent différentes expériences d'implications dans des projets de recherche et d'expertises d'accompagnement des politiques de recherche et d'innovation au Cameroun [TEM 17].

4 MINADER : Ministère de l'agriculture et du développement rural. IITA : International Institut on Tropical Agriculture. 


\section{Dynamique du système d'innovation du secteur cacao au Cameroun}

Les orientations de politique agricole au Cameroun visent à faire passer l'agriculture d'un stade « artisanal» vers un stade plus moderne ou dit de «deuxième génération ». Dans le cas de la production cacaoyère un premier plan de relance datant de 2002 a été élaboré suivi d'un second courant de 2014 à 2020 [CTS 14]. Le nouveau Document de Stratégie nationale de développement 2020-2030 (SND30) projette une production de 640000 tonnes de cacao d'ici 2030. Les deux plans de relances comportaient différentes actions polarisées par l'accroissement des rendements favorisant le développement de l'utilisation de plants de variétés améliorées issues des résultats de la recherche qui ont été reprise dans la SND30. L'innovation dans le secteur cacaoyer reste ainsi historiquement polarisée par deux composantes complémentaires.

D'une part, celle de l'amélioration du matériel végétal à croissance précoce et à hauts rendements ${ }^{5}$, comme les premières variétés hybrides introduites à dominante de Forastero et Trinatario [MFE 17]. Une vingtaine de variétés issues de sélection réalisée en station de recherche dans les années 60 par l'Institut de Recherche Agricole pour le Développement (IRAD) sont diffusées à partir des champs semenciers de la SODECAO. Cette dynamique de création variétale s'est estompée au début des années 90 avec le désengagement de l'Etat des activités de production.

Par ailleurs au regard de la relance actuelle, la demande annuelle potentielle de plants améliorés est estimée par la Société de développement du Cacao (SODECAO) à 100 millions alors que la production actuelle cumulée n'est que de 7 millions ${ }^{6}$.

D'autre part, la deuxième composante est celle du transfert des connaissances techniques permettant d'améliorer la gestion des cacaoyères pour lutter contre les principales maladies et ravageurs (pourriture brune et capsides) et optimiser la nutrition des cacaoyers. De manière récente et transversales se mettent en œuvre de stratégies d'améliorations de la qualité globale permettant de répondre à l'évolution des contraintes d'accès aux marchés internationaux [NLE 20] concernant les résidus de pesticides [GAL 20] ou les conséquences sur la déforestation [CAR 20].

\subsection{L'évolution de la production de connaissances scientifiques sur le cacao}

Avant la fin des années 2000, les publications scientifiques sont dominées par la thématique de la résistance génétique du cacao contre les maladies puis les ravageurs (après 2008). A partir de 2007, les publications vont se diversifier sur l'agroforesterie et plus largement les systèmes de culture. Cette diversification est fortement liée au lancement du Dispositif en Partenariat (DP) sur l'agroforesterie $^{7}$, qui a influencé l'orientation des recherches. Malgré cette diversification des connaissances, deux remarques méritent d'être soulignées. La première est relative à une faible présence des Sciences Humaines et Sociales (SHS) dans les publications scientifiques, notamment justifiée par l'absence de référencement des connaissances produites dans la base de données de Web of science ${ }^{8}$.

La deuxième révèle une faible connexion entre le contenu des projets de relance de la production soutenus par les bailleurs de fonds, pouvoirs publics et les nouvelles productions de connaissances

5 Environ 40 à $50 \%$ du verger a été planté avant 1950 et le rendement moyen est de $400 \mathrm{~kg} / \mathrm{ha}$.

6 Ce cumul intègre la production des plants de l'ensemble des intervenants publics et privés qui assurent la multiplication du matériel végétal et sa distribution / commercialisation dans toutes les zones de production.

7 Le DP Agroforesterie Cameroun est une plateforme de recherche créée en 2005 regroupant le Cirad et plusieurs instituts de recherche et d'enseignement supérieur camerounais et internationaux.

8 II faut noter qu'il existe de la littérature grise sur les aspects économiques et sociaux, produite par des chercheurs en sciences humaines et sociales et des publications dans des revues non référencées par la base Web of Science. 
scientifiques portant sur l'agroforesterie et la durabilité dans les systèmes cacaoyers. En effet le contenu des plans relance de la production est focalisé par l'accroissement de la production par les rendements, à partir d'un modèle «science push» polarisé par l'usage d'intrants dont principalement les nouvelles variétés. Ils ne référencent pas ou peu des accompagnements qui permettraient d'activer d'autres ressources potentielles des socio-écosystèmes comme le microbiote, les capacités d'innovations endogènes, ou surtout les relations de complémentarités entre cultures au sein des systèmes forestiers qui conduirait à intégrer la multifonctionnalité spatio-écologique : alimentaire [TEM 97], écologique, forestière [SEG 19], sanitaire de la production de cacao comme une ressource de la compétitivité.

\subsection{Une gouvernance institutionnelle de l'innovation très administrée}

Dès 1945, sous administration coloniale, l'accompagnement des planteurs est confié à des organismes techniques [CHA 66]. Ce dispositif d'accompagnement repose sur trois piliers : 1) l'amélioration des méthodes culturales, 2) le développement du matériel végétal et 3) la maîtrise des maladies (pourriture brune des cabosses, mérides et capsides). La création de la station de recherche de Nkoemvone ${ }^{9}$ en 1949, fixe le cadre de la recherche scientifique future. Cette structure deviendra plus tard la station spécialisée de l'IRAD, creuset de l'amélioration et de la multiplication variétales sur le cacaoyer.

L'objectif d'accroissement de la production s'appuyait sur plusieurs structures publiques au cours du temps. Le « Secteur Expérimentale de Modernisation des cacaoyères » (SEMAC) créé en 195354, était constitué de plusieurs « postes de paysannat » avec pour mission d'assurer l'encadrement et la vulgarisation. Les « Services de l'agriculture », ancêtres du ministère de l'agriculture renforçaient les missions d'encadrement des planteurs à travers les «Postes agricoles». La Société de Développement du Cacao (SODECAO) créée en 1974 a hérité du système organisationnel et des missions du SEMAC. Une Direction de la lutte phytosanitaire anticapsides (LPAC) complétait ce dispositif public.

La cartographie du réseau d'acteurs qui structurent la filière administrée révèle ainsi une concentration de l'action institutionnelle sur le segment de la production (Figure 1). Ce choix peut se justifier par l'objectif d'accroissement de la production dans un contexte de forte concurrence entre pays producteurs. En outre, l'action publique camerounaise dans le domaine de la production était financée par deux sources issues des mécanismes nationaux de régulation de la filière qui ont conduit à la création de la «Caisse de Stabilisation» dès 1955, et de «l'Office National de Commercialisation des Produits de Base »(ONCPB) en 1976 d'une part; et internationaux de stabilisation des exportations (Fonds STABEX, 1975-1999) d'autre part. La commercialisation intérieure et l'exportation, bien que théoriquement aux mains du secteur privé était contrôlée par le secteur public [LAP 92 ; FOL 03]. 


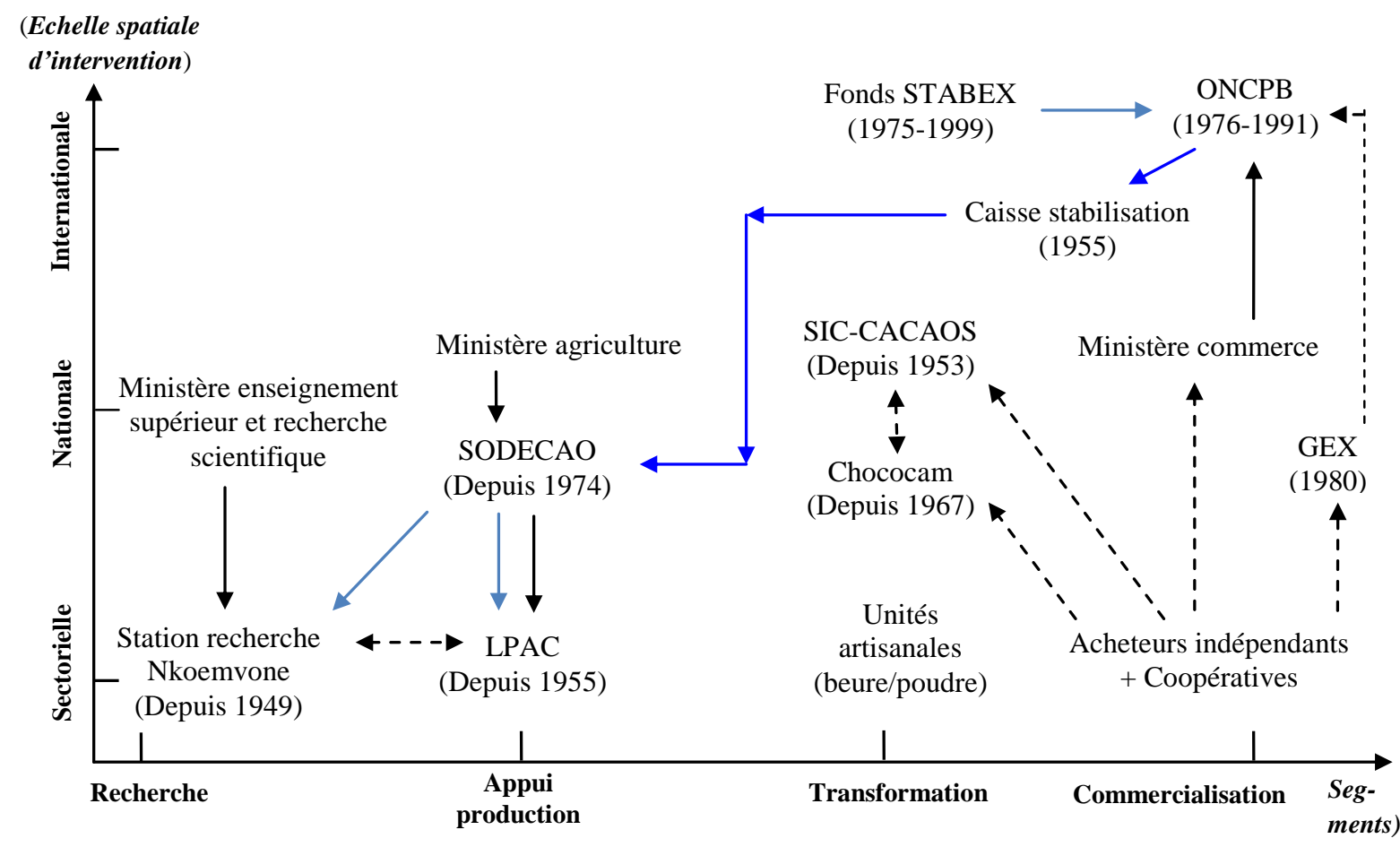

Légende :

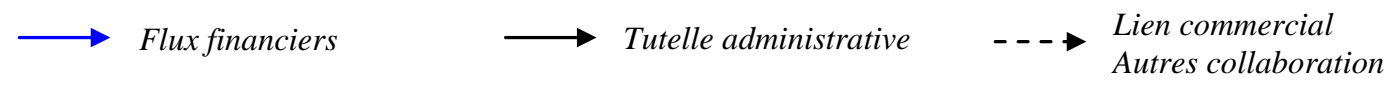

Figure 1. Matrice du système acteurs du secteur cacao administré

Cette cartographie traduit la volonté publique de spécialiser et d'assurer la complémentarité entre les différents organismes composant le sous-système d'appui à la production dans la chaîne de valeur. Cette complémentarité a en outre constitué un important vecteur de transfert de connaissances scientifiques et techniques aux planteurs (mise en culture, entretien des plantations, lutte contre les maladies, récoltes, séchage et stockage) à travers la SODECAO bras séculier de l'Etat. Ce sous-système intégrait également une dynamique territoriale multi scalaire de structuration productive et marchande (sectorielle, nationale et internationale), correspondant respectivement aux zones de production, points d'achat aux planteurs et marchés internationaux. Ce contrôle de l'espace cacaoyer à différentes échelles a permis de constituer de façon efficace une base statistique fiable sur la production (effectifs de planteurs, superficies cultivées, tonnages) et la commercialisation intérieure et internationale.

\subsection{La « libéralisation » : un vecteur de transformation sectorielle de l'innovation sur cacao}

La réforme du secteur cacaoyer à la fin de la décennie 1980 a diversifié le système-acteurs (Figure 2). Elle a été marquée par le désengagement de l'Etat au niveau des structures productives à la base et de l'encadrement du réseau de commercialisation interne et à l'exportation. Quatre segments sectoriels spécifiques (recherche, appui à la production, transformation et commercialisation) ont émergé de cette réforme qui a brisé le monopole de la SODECAO. Ainsi, sa mise en veille par la suppression des subventions publiques (1987-2001) a conduit à transférer certaines de ses missions au ministère de l'agriculture [encadrement des planteurs, multiplication et distribution du matériel végétal, protection des vergers]. Malgré la réhabilitation récente de la SODECAO, la plupart de ses missions restent assurées par des projets ministériels.

L'appropriation de ces missions interroge l'efficacité du suivi statistique sur la production, l'expertise et les infrastructures adéquates pour le renouvellement du verger et la protection contre les maladies. Dans le cadre de la lutte anticapsides précédemment menée par la SODECAO, la 
constitution d'un fichier planteurs structurait la construction d'une base statistique nationale. Malgré sa réhabilitation, le fichier de la SODECAO couvre moins de $80 \%$ de la totalité des bassins de production au niveau national. Les statistiques mobilisables sur la production nationale proviennent d'une diversité de sources (Ministère de l'agriculture, SODECAO, ONCC, GEX). Elles comportent des écarts quelquefois significatifs, même si elles peuvent être recoupées à travers les exportations en intégrant la proportion transformée au niveau national. La transformation (environ $20 \%$ de la production) est contrôlée par les Sociétés industrielles des cacaos (SIC-CACAOS) filiale du Groupe Barry Callebaut et les Chocolateries et confiseries du Cameroun (CHOCOCAM). Dans le cadre de la relance de la filière, la construction d'une dizaine d'unités de transformation prévoit l'accroissement de la proportion transformée à près de $30 \%$ de la production actuelle.

La réforme du secteur a ainsi redéfini les responsabilités des différents intervenants dans la filière. Elle désengage les organes étatiques de la commercialisation. L'Etat par le biais de « 1'Office National du cacao \& Café » (ONCC), qui remplace l'ONCPB en 1991, assure les fonctions de régulateur. Les acteurs clés de la filière (producteurs, acheteurs-usiniers, transformateurs industriels et professionnels de l'exportation) sont regroupés depuis 1991 au sein du Conseil Interprofessionnel du Cacao et du Café (CICC). L'exportation du cacao est gérée exclusivement par le secteur privé représenté par le Groupement des Exportateurs de cacao-café (GEX) créé en 1980 et regroupant une soixantaine de membres. Un système de financement des acteurs institutionnels et des projets publics sur les filières cacao et café a par ailleurs été mis en place en 2006, par la création du Fonds du Développement du Cacao et du Café (FODECC).

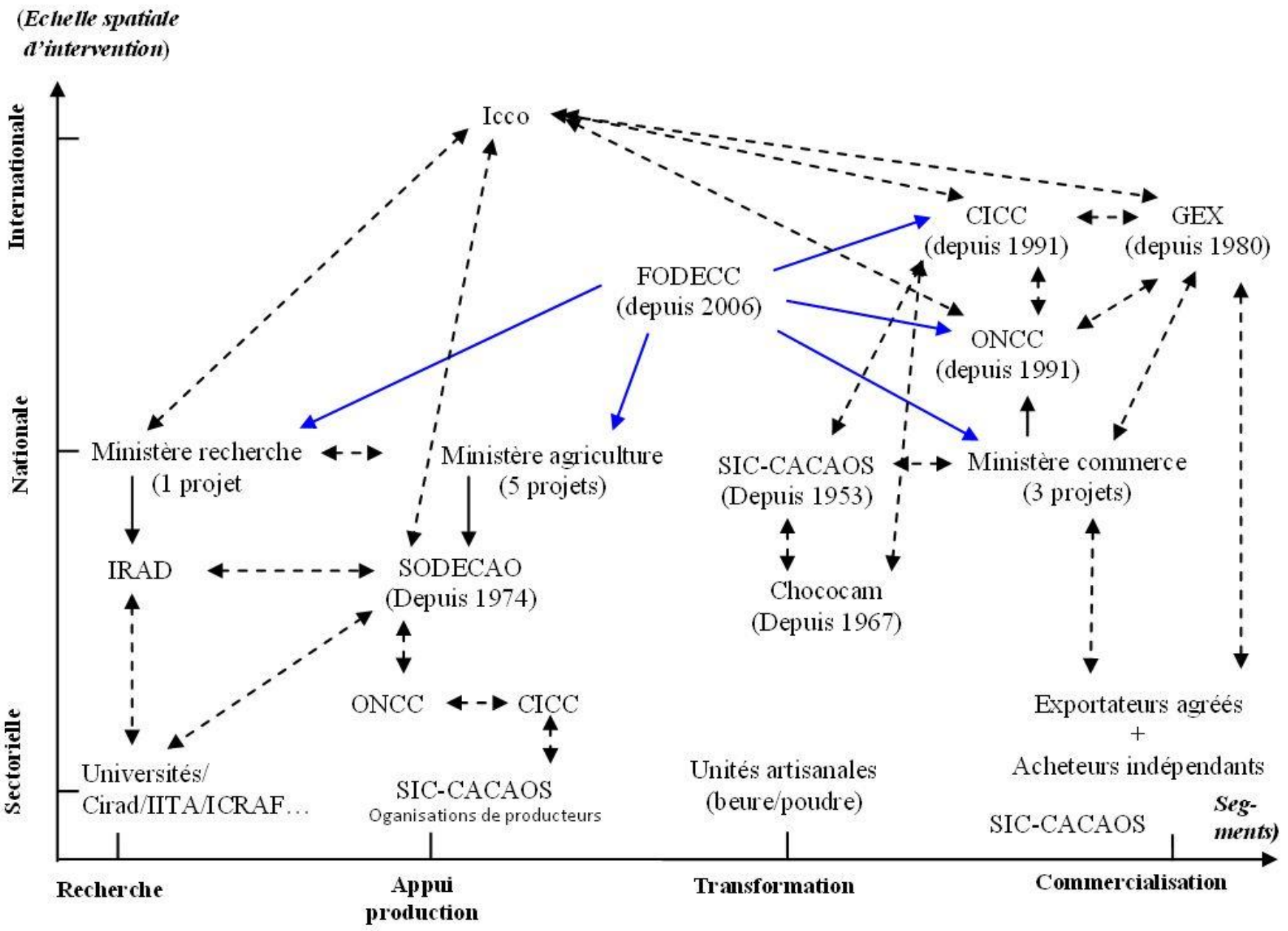

Légende :

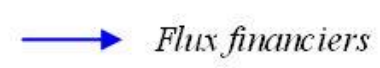

Figure 2. Cartographie des institutions structurant le système sectoriel d'innovation 
La libéralisation a ainsi entrainé une densification des intervenants et une complexification des rapports entre les acteurs de la régulation étatique et du secteur privé. Le cacao est au cœur d'antagonismes entre organismes publics d'une part, et entre structures publiques et privées d'autre part, interrogeant les conséquences sur la cohérence des politiques de recherche, d'innovation et d'accompagnement du secteur.

Une fonction clé au cœur de cet antagonisme concerne la production et la distribution de plants de variétés améliorées. Cette fonction est aujourd'hui fragilisée par des individualités impliquant les organes de l'Etat (Ministère de l'agriculture -Minader-), SODECAO, Fodecc), les organismes et entreprises privés (CICC, SIC-CACAOS), les institutions de recherche (IITA, IRAD, Cirad) et les élites politiques. L'analyse des données mobilisables des trois principaux intervenants (Sodecao, Minader et IITA) montre des variations inter annuelles qui interrogent la consolidation du rôle moteur de la SODECAO en tant que bras séculier de l'Etat. Cependant, d'un point de vue spatial, la distribution de plants améliorés reflète une demande forte des producteurs pour le rajeunissement de leurs vergers dans les bassins cacaoyers traditionnels et pour le développement des fronts pionniers notamment dans le Mbam (Figure 3).

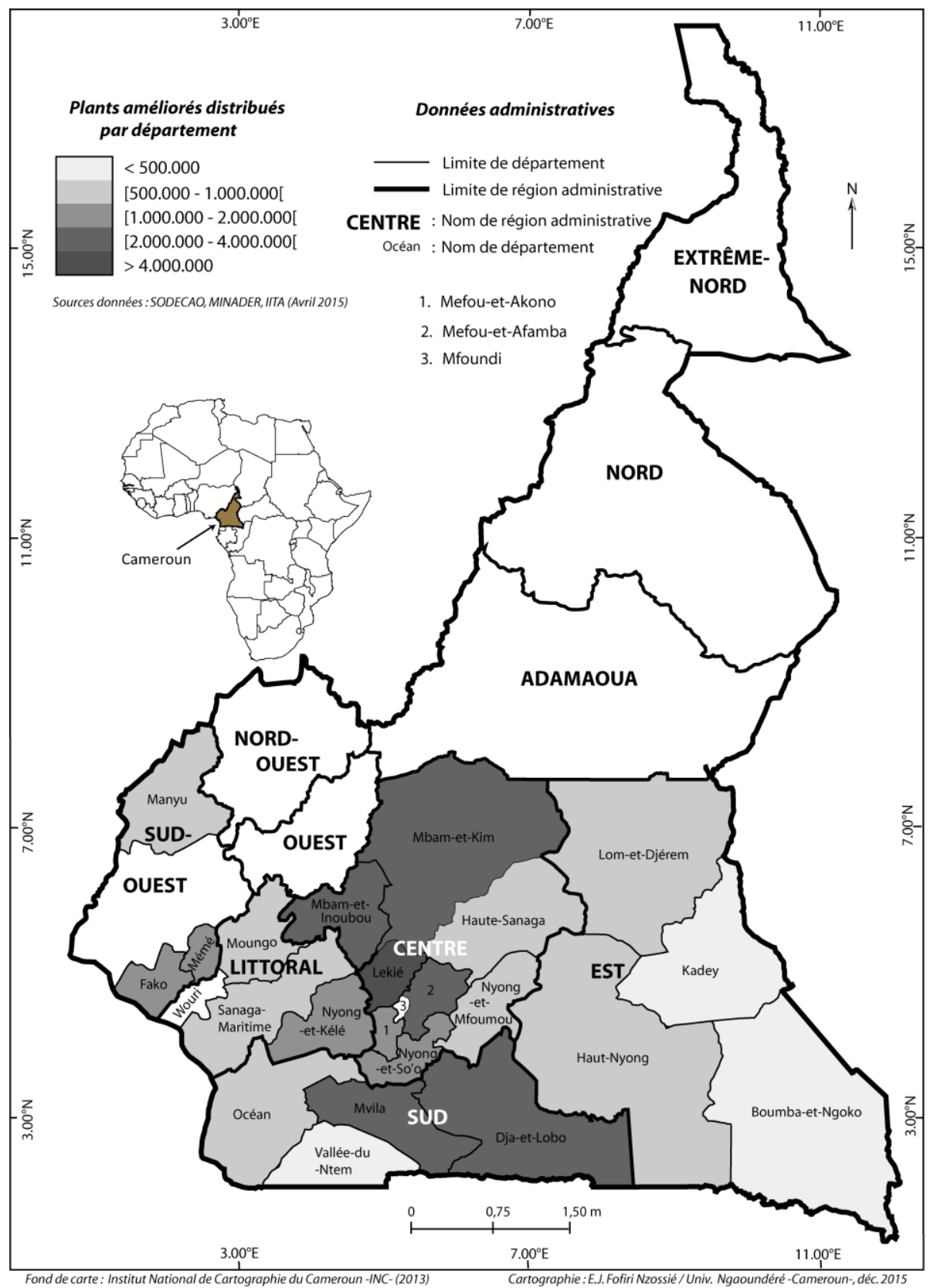

Figure 3. Distribution de plants améliorés dans les bassins de production au Cameroun (2009-2013) 
Un autre ensemble de fonctions clés lié à la durabilité est celui de la certification [NLE 20] qui a commencé à se mettre en place au Cameroun à partir des années 2010 par différentes normes. L'objectif de l'évolution des cahiers des charges étant principalement de diminuer la déforestation. Il se traduit par la recommandation de bonnes pratiques agricoles basées parfois sur une utilisation plus forte intensification en pesticides. Les acteurs impliqués dans la certification sont principalement des ONG, des organisations et coopératives de producteurs et des entreprises privées (exportateurs) [NLE 20].

\section{Quelles conséquences sur les politiques de recherche et d'innovation du secteur?}

\subsection{Une gouvernance variable du système sectoriel d'innovation questionné par l'agroforesterie}

L'analyse du système sectoriel cacaoyer au Cameroun montre deux grandes visions du développement de ce secteur. La première est portée par la prédominance de l'« acteur-recherche » dans le système d'innovation. Les recherches sur le cacao ont été par le passé très orientées sur les aspects biophysiques, les connaissances de base sur les aspects économiques, institutionnels et organisationnels étant peu considérées ou focalisées sur des analyses micro-économiques (Leplaideur, 1995). Cette polarisation techniciste ne permettait pas ou peu de référencer les contraintes méso et macro-économiques à l'innovation de natures sociales, économiques, organisationnelles et institutionnelles.

En revanche depuis quelques années l'orientation récente de la recherche agronomique sur l'agroforesterie et l'intensification de «systèmes de culture associés avec le vivrier » élargit les bases de connaissances et les processus d'innovations qu'elles permettent de documenter. Ainsi les travaux sur les conditions de mise en valeur des sols associant simultanément ou séquentiellement des arbres, des productions vivrières ou des animaux impliquent de resituer la production de cacao dans la dynamique globale du système agraire. L'idée est d'améliorer les interactions écologiques et économiques entre les formes de mise en valeur associées. Cette approche privilégie la recherche des conditions de maintien de la productivité du sol des terres cultivées plutôt que celle de la mise en exploitation de nouvelles terres, en même temps qu'elle diversifie le modèle technique sur les différentes modalités d'accroissement de la production. Elle permet de rendre plus conciliable la santé des plantes, des écosystèmes et des agriculteurs dans un moindre usage aux intrants de synthèses.

D'un autre côté se situe la vision portée par différents acteurs institutionnels et les entreprises aval de la filière, avec une volonté d'accroitre la production par la productivité à partir des résultats de recherche dans un modèle d'innovation basé sur le «science push ». Cette vision s'explique par trois dimensions. La première est celle d'une certaine nostalgie de la période de prospérité d'encadrement étatique face à l'inertie institutionnelle actuelle. La deuxième est celle de la tendance à la hausse des prix du cacao sur le marché international. La troisième est celle des incitations données par les bailleurs de fonds internationaux. Dans ce contexte de relance la production est en grande partie pilotée par les prix internationaux et l'offre en plants et semences issus de la recherche se réalise principalement par l'extension des fronts pionniers de mises en valeur des forêts ou parfois des savanes (à l'exemple de la plaine du Mbam).

La politique sectorielle de pilotage de l'innovation par la diffusion de variétés à travers ses organes spécialisés (SODECAO, ONCC, FODECC), mais également des organismes et entreprises parapublics (CICC) et privés (GEX, SIC-CACAOS, CHOCOCAM) est en soit interrogée par les nouvelles recherches qui diversifient les conditions de conception de l'innovation. En effet l'adéquation de ces variétés aux objectifs d'optimisation des écosystèmes complexes dans le cadre de systèmes associés, adaptés à des situations localisées et anticipant les besoins d'adaptation au 
changement climatique et à l'épuisement de la rente de fertilité de sols sur une plante pérenne, interpelle la pertinence des orientations en cours.

La relance du secteur est un enjeu de développement de l'agriculture camerounaise. Cet enjeu est porteur d'intérêts économiques et financiers forts particulièrement dans les segments de production et de commercialisation. Le cacao est une des principales sources de valeur pour les entreprises du secteur agro-alimentaire globalisées et celui du secteur agro-chimique (exportateurs, importateurs, chocolatiers, phytosanitaires,...). Le poids institutionnel de ces acteurs dans les orientations technologiques en cours est majeur au regard des déconnexions observées entre la structure des actions de développement en cours et de l'offre de recherche.

Dans le segment de la production qui concentre les projets de relance, on observe peu de coordination et parfois une compétition entre des organismes techniques de vulgarisation des résultats de la recherche, conduisant parfois à une duplication des rôles et à un manque de coordination. L'existence de cette compétition est expliquée par l'historique de la construction du dispositif institutionnel d'encadrement de la production qui dans le processus de libéralisation, a vu se superposer de nouveaux acteurs sur les mêmes fonctions, particulièrement sur celle de distribution des plants améliorés, mais de plus en plus d'encadrement des producteurs. La coordination pourrait porter dans un premier temps sur la réunification $d u$ système d'information statistique sur le suivi du renouvellement des vergers. En l'état actuel, la diversité des sources statistiques crée une opacité sur le bon calibrage des besoins d'approvisionnement en plants, mais aussi un risque pour appréhender les solutions en cas de crise sanitaire touchant le verger cacaoyer. Enfin l'intégration de suivi concernant l'impact de nouvelles variétés sur le plan environnemental et social semble tout aussi importante pour une bonne orientation des programmes d'amélioration génétique de la recherche. Cela aux regards des objectifs de la durabilité systémique et pas uniquement des rendements obtenus en station expérimentale.

\subsection{Un enjeu de gouvernance de l'innovation par l'hybridation des connaissances scientifiques et profanes}

Les connaissances, pas uniquement de nature technique, peuvent être ou non spécifiques au secteur. Elles sont véhiculées par des processus d'apprentissages et d'expérimentation au sein du secteur dans la mesure où elles sont utiles, utilisables et utilisées. Elles peuvent provenir tant de la recherche que des acteurs non académiques (Entreprises privés, Société civile, Communautés rurales). Si les connaissances sont présentées comme un élément clé des SSI, il faut préciser que ce n'est pas le stock de connaissances en soit qui est important, mais plutôt leur circulation entre les acteurs du système et les processus d'apprentissage collectif qui mettent à l'épreuve leur appropriation et usages dans la mise en œuvre de changements [BRE 14]. Cette circulation nécessite que ces connaissances soient mises en traduction pour différentes communautés d'acteurs donc transformées et relayées par des acteurs intermédiaires appelés parfois «brokers »[KLE 09; KLE 12]. Initialement cette traduction était portée par des organismes techniques sous tutelle étatique, mandatés pour la promotion des résultats de la recherche. Depuis les politiques de libéralisation, ces structures d'intermédiation se sont diversifiées via les projets soutenus par la coopération internationale sur le secteur privé, les ONG principalement. Ces derniers se retrouvent parfois en compétition non seulement entre eux, mais aussi avec les organismes publics. Pourtant malgré ce renouvellement des structures d'intermédiation, on note un essoufflement de la logique de transfert dont rend potentiellement compte la faible adoption par les producteurs des technologies proposées par la recherche [DUC 14 ; FOF 15].

Les technologies proposées soit ne répondent pas ou plus aux besoins des producteurs et du marché, soit leurs usages se révèle trop contraignants ou risqués au regard des conditions d'accès aux ressources des agriculteurs, de leur vulnérabilité et de son accroissement (crises climatiques, sanitaires). Cet essoufflement explique que les approches en termes de systèmes d'innovation 
tentent de promouvoir des modèles d'innovations plus collaboratif fondés plus sur l'hybridation des bases de connaissances et d'expérimentation scientifiques et experimentielle des agriculteurs que sur de simples transferts technologiques. Ces dernières se révèlent d'autant plus importantes dans les pays en développement où les systèmes d'information, formations formelles sont peu accessibles à des agriculteurs en situation précaires et où l'accès aux connaissances codifiées et à leurs produits implique des conditions réservées qu'a des situations particulières d'élites. Ainsi, l'amélioration des systèmes d'innovation à travers la prise en compte des connaissances issues de l'expérience, des apprentissages collectifs et qui sont souvent non codifiées par des fiches techniques ou des articles de recherches représente un enjeu important pour le renforcement des SSI.

\subsection{Une reconfiguration potentielle des modèles d'innovation dans le secteur du cacao}

Le modèle linéaire d'innovation de transferts technologiques des variétés à haut rendement de la recherche aux producteurs sous-tend pour partie encore de manière dominante, les politiques d'innovation structurées par les projets de relance actuels de la production soutenus par les pouvoirs publics et les bailleurs de fonds. Ces projets continuent de se concentrer sur la diffusion de nouveaux inputs (variétés, pesticides). Les nouveaux modèles d'innovation plus systémiques qui renvoient à une approche plus complexe supposent des orientations politiques différentes [EDQ 12]. Ils impliquent une meilleure articulation entre acteurs dans la conception des politiques d'innovation [TEM 20] que structurent les projets de développement soutenus, les connaissances que proposent la recherche mais également les bases de connaissances et d'expériences des agriculteurs liées à des actions collectives adossées à la résolution de contraintes localisées.

Cet élargissement impose de ne plus se focaliser sur une perspective unique de réponse à un besoin d'accroissement rapide de la production porté d'ailleurs au niveau global par différentes instances, voire par la demande des firmes globalisées. Il impose d'intégrer des questionnements relatifs à la durabilité environnementale et sociale de la production [CAR 20] et structure trois propositions de recommandations.

La première est le renforcement de la coordination des acteurs impliqués dans le secteur cacaoyer par la clarification, voire la redéfinition de leurs rôles pour éviter des chevauchements sur les mêmes fonctions et auprès des mêmes cibles. Dans le contexte actuel de la relance, cette coordination peut s'organiser au sein d'une cellule inter-institutionnelle tel que la Cellule Technique de suivi et de Coordination des filières cacao et café. Elle impliquerait par exemple de recadrer le rôle des institutions de recherche dans la production de connaissances et des outputs (matériel végétal amélioré, itinéraires techniques), les entreprises agro-industrielles dans la formulation des besoins d'amélioration de la qualité et l'accompagnement de la dynamique de production (financement des inputs).

La seconde proposition porte sur le support de l'élargissement de la capacité de la recherche à prendre en compte la spécificité des contraintes (organisations sociale, économique, problématiques foncières) qui structurent la durabilité (vecteur de compétitivité dans le temps long). Cela intègre des questions sur l'adaptation des choix techniques aux besoins d'optimisation des ressources de l'écosystème, de réduction de la dépendance à l'industrie chimique, d'adaptation au changement climatique et enfin d'amélioration des conditions de répartition de la valeur pour les catégories d'acteurs les plus vulnérables dont font partis les planteurs. Si on note une croissance importante de la production de connaissances scientifiques sur les interactions entre plantes au sein de systèmes agro-forestiers [JAG 11], sur l'épidémiologie des maladies au regard des différents écosystèmes [HAL 05], sur l'impact du changement climatiques [MAG 14], l'utilisation de ces connaissances scientifiques par les organisations en charge de la relance technique de la production de cacao reste à confirmer. Il est donc nécessaire de considérer la mise en place d'une structure nationale de dialogue sur l'intermédiation entre les résultats de la recherche et les acteurs de la production afin 
d'évaluer clairement et de structurer les infrastructures nécessaires pour répondre aux besoins des acteurs de la production.

La troisième proposition consiste à densifier l'investissement dans l'accompagnement de la professionnalisation des producteurs, la structuration des acteurs privés au cœur de l'intermédiation entre les exportateurs et les planteurs, et le renforcement de leurs capacités managériales afin de garantir le niveau de revenu des producteurs dans leurs relations de mise en marché des fèves.

\section{Conclusion}

A travers la mobilisation du cadre d'analyse des SSI, nous avons qualifié les trajectoires d'innovations dans le secteur cacaoyer au Cameroun en identifiant les conditions institutionnelles, historiques et politiques de production des connaissances et des technologies. L'usage exploratoire de ce cadre utilisé est cependant resté partiel au regard des dispositifs d'information et connaissances mobilisables. Les résultats documentent néanmoins différentes options de reconfigurations des politiques publiques d'accompagnement et de recherche pour une cacaoyère qui intègre dans la recherche de compétitivité polarisée par les rendements [LES 19], d'autres paramètres liés à la durabilité des socio-écosystèmes productifs. Ils soulignent actuellement un réel besoin de coordinations et de réorganisation des dispositifs institutionnels d'encadrement de la production. Le maintien d'objectif classique de polarisation de la recherche et l'innovation sur l'accroissement quantitatif de la production (augmentation des rendements, de superficies plantées) ne sont pas suffisants pour une compétitivité durable. Les cycles du marché mondial du cacao connaissent des retournements dans le temps. L'intégration de variables relatives à l'optimisation des ressources des écosystèmes, la réduction des pesticides, la compatibilité aux cultures alimentaires, l'adaptation aux effets des changements climatiques dans les projets de développement de la cacaoculture sont des orientations constructives pour une cacaoyère durable sur des créneaux de qualité, qui accroissent la captation de la valeur dans les filières mondiales. L'institutionalisation publique des dispositifs de certification du cacao est un levier de gouvernance des innovations techniques qui sont mobilisées dans les filières cacao. Elle impose dans l'élaboration des cahiers de charges qui sous tendent pour ces certifications de promouvoir de bonnes pratiques agronomiques qui permettront de réduire réellement l'usage des pesticides. Elle impose de nouvelles formes de collaborations entre les différents acteurs du système $d$ 'innovation et remet en question les conceptions linéaires de l'innovation basées sur la simple diffusion de nouveautés techniques mises au point dans les laboratoires de recherche. .

\section{Bibliographie}

[ALA 96]. AlaRY V., Incertitude et prise de risque en période d'ajustement: le comportement des producteurs de cacao du Cameroun avant et après 1994, Thèse de Doctorat, Université de Paris-Panthéon Sorbonne, France, 1996

[BIS 14] Bisson P., ALAMI S., TEMPLE L., Etude sur l'économie de la recherche au Cameroun. Programme d'Appui à la Recherche. Ministère de la recherche scientifique et de l'innovation, Cirad, Yaoundé, Cameroun, 2014

[BRE 97]. BRESCHI S., MALERBA F., "Sectoral innovation systems: technological regimes, Schumpeterian dynamics and spatial boundaries", in Systems of Innovation: Technologies, Institutions and Organizations Ed. C Edquist (Frances Pinter, London), 1997

[BRE 14]. BRETTE O., "La valorisation économique de la recherche scientifique". Principes d'économie de l'innovation. S. Boutillier, J. Forest, D. Gallaud et al., Bruxelles 8, 2014

[CAR 20]. CARIMENTRAND A., Cacao. Etat des lieux sur la déforestation et les standards de durabilité. Rapport d'étude pour le CST Forêts de l'AFD, Cirad Montpellier, 2020

[CAS 17].CASADELLA V., TAHI S., TEMPLE L., "Créativité et politiques d'innovation". Journal of Innovation Economics \& Management, 53 2017/2, 2017.

[CHA 66]. Champaud J., "L’économie cacaoyère du Cameroun". Cah. Orstom, 1966 
[CTS 14]. CTSCCC, Plan de relance et de développement des filières cacao et café du Cameroun Horizon 2020, Cellule technique de suivi et de coordination des filières cacao et café (CTSCCC), Services du premier ministre, secretariat général, Cameroun, 2014

[DUC 14]. Duchamp M., Temple L. And Ndoumbe Nkeng M., "Cadrages méthodologiques de l'analyse bibliométrique". Etude sur l'économie de la recherche au Cameroun, Rapport d'expertise, Minresi-Cirad, Yaoundé, 2014

[EDQ 12]. Edquist C. And Zabala-IturRiagagoitia J.M., "Public Procurement for Innovation as mission-oriented innovation policy". Research Policy 41(10), 2012.

[FOF 15]. FofiRi NZOSSIE E.J., TEMPLE L. AND NDJOUENKEU R., "La contribution de la recherche universitaire à la formation d'un système sectoriel d'innovation agro-alimentaire au Cameroun". Innovations 47(2), 2015.

[FOL 03]. FOLEFACK DP., Analyse socio-économique de la commercialisation du cacao en zone forestière du sud Cameroun, Diplôme d'Etudes Spécialisées en Economie et Sociologie rurales, Faculté Universitaire des Sciences Agronomiques de Gembloux, Belgique, 2003.

[Gal 20]. Galani Y.J.H., Houbraken M., Wumbei A., GJeugap J.F., Wumbei A., Fotio D., Gong Y.Y. \& Spanoghe P., "Monitoring and dietary risk assessment of 81 pesticide residues in 11 local agricultural products from the 3 largest cities of Cameroon". Food Control, 2020.

[GEE 04]. GEELS F.W., "From Sectorial Systems of Innovation to Socio-Technical Systems: Insights about Dynamics and Change from Socilogy and Institutional Theory". Research Policy, 33 (6-7): 2004

[HAL 05]. HALl A., "Capacity development for agricultural biotechnology in developing countries: an innovation systems view of what it is and how to develop it". Journal of International Development 17(5), 2005.

[JAG 11]. JAGORET P., Michel-Dounias I. AND MALEZIEUX E., "Long-term dynamics of cocoa agroforests: a case study in central Cameroon". Agroforestry systems 81(3), 2011

[KLE 09]. KLERKS L., HALl A. AND LEEUWIS C., "Strengthening agricultural innovation capacity: are innovation brokers the answer?". International journal of agricultural resources, governance and ecology 8(5), 2009.

[KLE 12]. KLERKK L., SCHUT M., LEEUwIS C. AND KILELU C. "Advances in knowledge brokering in the agricultural sector: towards innovation system facilitation". IDS Bulletin 43(5), $2012 \mathrm{a}$.

[LAP 92]. LAPORTE B. Les reformes des systèmes de commercialisation et de stabilisation des café et cacao au Cameroun et en Côte-d'Ivoire, CERDI, 1992

[LES 19]. LESCUYER G., Boutinot L., Goglio P. \& BASSANAga S., Analyse de la chaine de valeur du cacao au Cameroun, CIRAD, Wagenigen University \& Research. https://agritrop.cirad.fr/595017/, 2019

[LEP 95]. LEPLAIDEUR A., "Strategies paysannes autour des systèmes cacaoyers et vivriers en zone centre-sud forestière camerounaise", Etats, Developpement, Paysan s: Actes du colloque CIRAD-MESRU, 1995

[MAG 14]. Magne A.N., Nonga N.E., Yemefack M., Robiglio V., 'Profitability and implications of cocoa intensification on carbon emissions in Southern Cameroun', in Agroforestry systems, 88 (6), 2014

[MAL 05]. MALERBA F., "Sectoral systems of innovation: a framework for linking innovation to the knowledge base, structure and dynamics of sectors". Economics of Innovation and New Technology 14(1-2), 2005

[MFE 17]. Mfeck Eyenga L.B., Kamdem C., Temple L., Mathé S., "Rendements et mécanismes d'adoption du matériel végétal amélioré : le cacao au centre-Cameroun". Tropicultura, 35 (2), 2017

[MIN 18]. Minkoua NziÉ JR., TEMPLE L., L'offre de recherche converge-t-elle vers les besoins du secteur agroalimentaire au Cameroun? Une analyse par la bibliographie. Cah. Agric. 27: 25008, 2018

[NLE 20]. Nlend NKotT AL., MAthÉ S., TeMPle L., Analyse multi-niveau des freins à l'adoption de la certification du cacao au Cameroun. Economie Rurale, 370, 2020

[REP 21]. RÉPUBLIQUE DU CAMEROUN. Stratégie de développement du secteur rural/ plan national d'investissement agricole SDSR/PNIA (2020 - 2030), 2021

[SEG 19]. SEGHIERI J., HARMAND J.M., Agroforesterie et services écosystémiques en zone tropicale. Versailles : Quae, 2019,. ISBN 978-2-7592-3059-4

[TEM 20]. Temple L., CASSAdella V., Politiques et modèles d'innovation en Afrique, Technologie innovation : Vol 5, n³. http://www.openscience.fr/Politiques-et-modeles-d-innovation-en-Afrique, 2020 
[TEM 17]. Temple L., Machikou Ndzesop N., Fongang FouePe G.F., Ndoumbe NkenG M., Mathe S., « Système national de recherche et d'innovation en Afrique : éclairage du Cameroun. Innovation », Journal of Innovation Economics \& Management, Innovations 2 (53), 1997

[TEM 97]. TEMPLE L., FADANI A, «Cultures d'exportation et cultures vivrières au Cameroun : l'éclairage d'une controverse par une analyse micro-économique ». Economie Rurale, 1997

[TOU 14]. Touzard JM., Temple L., Faure G.,. TRiOMPhe B, "Systèmes d'innovation et communautés de connaissances dans le secteur agricole et agroalimentaire". Innovations, 43(1), 2014 